\title{
Dexterity Measure for Tendon Actuated Parallel Mechanisms
}

\author{
Ronald Kurtz and Vincent Hayward \\ McGill Research Center for Intelligent Machines \\ 3480 University Street \\ Montréal, Québec, H3A 2A7, Canada
}

\begin{abstract}
We look at a class of tendon actuated mechanisms. We show that the conventional dexterity measures used for the analysis of manipulators are not applicable to the tendon case because there are non-linear elements. We then develop a correct dexterity measure for this type of mechanism.
\end{abstract}

\section{Introduction}

Tendons are unidirectional actuators that are inherently nonlinear. As a consequence, any analysis of tendon systems are bound to be much more complex than ordinary bi-directional actuator mechanisms. Along with this, tendon transmissions are often complicated, requiring low friction pulleys to route the cables over joints and other structures as well as force sensors to monitor the cable tension. Design of the tendons themselves is an art, as they undergo high stress but must have a long life and be resistant to wear and stretching. Finally, the workspace of mechanisms driven by tendons may be smaller than those using conventional linear or rotary actuators.

Despite these facts there are many advantages to using tendon transmissions, and in some cases there is no other alternative. Most actuators such as DC motors are relatively bulky. De-locating these actuators can improve on the manipulator dynamics and structural properties. For multifinger hands a large number of joints are located in a small volume. There is simply not enough room to place the motors near the joints; we are forced to use some kind of flexible transmission system such as tendons. Salisbury (1988)

Manuscript received April 7, 1991. This work has been supported by NSERC, the Natural Science and Engineering Research Council of Canada, FCAR Les fonds pour la formation des chercheurs et l'Aide à la Recherche, Quebec, and IRIS the Institute for Robotics and Intelligent Systems. showed that when properly used, pretensioned cable drives have high stiffness and no backlash. This, combined with their small mass, low friction and geometric flexibility make them well suited for many applications.

In micro-actuation, magnetic servo levitation falls also under the scope of the analysis derived in this paper. This is because this type of actuator is unidirectional as electro-magnets can only attract their armatures (Tsuda, Nakamura, Higuchi 1990).

Several working tendon systems exist, most notably the human hand. Mechanical hands such as the UtahM.I.T. Dextrous hand (Jacobsen et. al., 1984) use tendons exclusively, but in antagonistic pairs. That is, each joint is controlled by two tendons acting in opposition, independent of the other joints. After suitable control has been introduced, each pair of tendons behaves as a single bi-directional actuator, thereby simplifying the kinematics. There are also applications of tendon transmissions in the field of force reflecting hand controllers for telemanipulation. This is due to the fact that tendons provide a zero backlash high efficiency environment essential for quality force transmission (Vertut 1976; McAffee 1988).

Certainly, for many applications tendons are better suited than other transmission systems, but as stressed previously the kinematic analysis is often more difficult. In the next section some fundamental questions will be addressed, such as what is the minimum number of tendons required, and how to ensure that the tension on the tendons remains positive.

\section{Kinematics of Tendons}

Tendons differ from other actuators as they can only support tensions and not compressive forces. Thus for the case of static forces we have additional constraints, namely to keep all the actuator forces positive. A sim- 
ilar problem occurs when an object is grasped by a hand. The fingertips can only exert positive forces on the object to constrain the grasp. This area has been well researched and has generated the following result (Lakshminarayana 1978):

Theorem: A rigid body with $n$ degrees of freedom can be completely constrained by $n+$ 1 point contacts.

For our purposes this can also be interpreted as $n+1$ tendons are needed to control an $n$ degree of freedom mechanism. It is easy to show why $n$ tendons are insufficient for this task. Consider the Jacobian transform relating joint forces $\mathbf{f}$ to external wrench $\mathbf{w}$ under conditions of static equilibrium.

$$
\mathbf{w}=\mathbf{J}^{T} \mathbf{f}
$$

If the robot is not at a singular configuration the $n \times n$ Jacobian will be invertible, and thus

$$
\mathbf{f}=\mathbf{J}^{-T} \mathbf{w}
$$

where $\mathrm{J}^{-T}$ is the inverse of the transpose of the Jaco bian. Say for some particular $\mathbf{w}$ all the joint forces are positive. If the wrench $-w$ is applied to the platform then all the joint forces must be negative. Tendons can not support negative forces, and thus $n$ tendons are not enough to control the mechanism independent of the applied wrench.

Now consider the case of $n+1$ tendons for the same mechanism. Here the Jacobian is not invertible, but using the pseudo-inverse we have:

$$
\mathbf{f}=\mathbf{J}^{+} \mathbf{w}+\lambda \mathbf{h}
$$

where $\mathbf{h}$ is a vector in the nullspace of the Jacobian, and $\lambda$ is a free parameter. As long as all the components of $\mathbf{h}$ are positive, $\lambda$ can be set sufficiently large to ensure that all the joint forces are positive, regardless of $\mathbf{w}$. Keeping the elements of $\mathbf{h}$ greater than zero is a necessary and sufficient condition for controllability of the mechanism. Qualitatively the extra tendon is needed to supply a bias force to bring all the joint forces to the positive range.

These additional constraints act to reduce the workspace of the mechanism. Consider the two tendon crank system in figure $1(b)$ derived from the piston example of figure 1(a). Here the crank can turn through a limited angle, whereas in the piston case there was no limit on the rotation.

We have just shown that tendon implementations have additional constraints that effect the kinematics

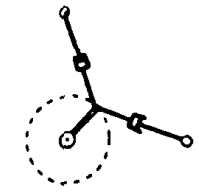

(a)

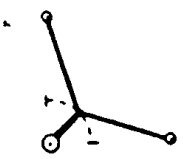

(b)

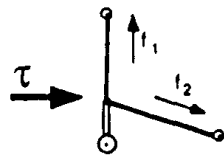

(c)
Figure 1: Crank mechanism driven by two pistons (a), and the corresponding tendon implementation $(b)$. The range of rotation of the tendon design is inherently limited (dashed line). At the edge of the workspace the tendons cannot resist even the smallest torque in the indicated direction $(c)$.

and workspace. It is not surprising that conventional dexterity measures are not applicable to this situation. A new dexterity measure for tendons will be proposed next section.

\section{Dexterity of Tendon Mechanisms}

Dexterity measures are crucial for finding optimal working points, postures, path planning and robot design. Therefore there is considerable motivation for investigating dexterity measures in the tendon case. Surprisingly this topic has received little attention in the research literature. Much work has been done on a related topic, that of finding optimal grasps but these results are often not directly applicable to robot dexterity. Kerr and Roth (1986a) select optimal grasp forces as those which maximize the distance from a set of inequality constraints. These constraints are derived from friction effects and limits on joint torques. The only constraint for a tendon is that the force remains positive, thus maximizing the distance from such a constraint results in an unbounded force. For magnetic servo levitated manipulators a method for optimizing the magnetic forces to reduce power consumption has been proposed (Tsuda et. al. 1989), but is dependent on the wrench applied to the object. As such it is not general enough for design. For the case of convex polygons, Markenscoff and Papadimitriou (1989) define 
optimal grasps as those with the smallest joint forces per unit wrench applied to the object. Similarly for the wrist mechanism, the tendon forces will be investigated as a means of quantifying dexterity.

Condition number is a common measure of dexterity, but it does not work for tendons. This can be seen by looking at the tendon-crank when it is at the limit of its workspace (figure $1(c)$ ). No amount of pulling at the tendons can resist even the smallest of torques in the indicated direction. Intuitively, this is a very poorly conditioned configuration. The dexterity does not reflect this fact, as the singular value at this location is non-zero. In fact, if pistons replaced the tendons then this configuration would be well conditioned.

A new dexterity measure will now be derived to deal with this problem. It is desirable to have a measure with the same physical significance as the condition number, giving an upper bound on the propagation of errors in the linear system. From Salisbury and Craig (1982):

$$
\frac{\|\delta \mathbf{f}\|}{\|\mathbf{f}\|} \leq k(\mathbf{J}) \frac{\|\delta \mathbf{n}\|}{\|\mathbf{n}\|}
$$

where $n$ represents the wrench of external forces, $f$ the set of actuator forces. As a measure of dexterity, the condition number is related to the accuracy of the mechanism in a specific configuration. It can also be thought of as a measure of sensitivity to external perturbations. If $\delta \mathbf{n}$ is a small disturbance, then the condition number gives an upper bound on the resulting relative change in actuator forces. Any $m \times n$ matrix $\mathbf{J}$ can be factored into the following singular value decomposition form:

$$
[\mathbf{J}]_{m \times n}=\left[\mathbf{U}^{T}\right]_{m \times m}[\mathbf{\Sigma}]_{m \times n}[\mathbf{V}]_{n \times n}
$$

where $U^{T}=1, \quad \mathbf{V V}^{T}=1$, and $\boldsymbol{\Sigma}_{i, j}=$ $\left\{\begin{array}{ll}\sigma_{i} & \text { if } i=j \\ 0 & \text { otherwise }\end{array}\right.$; with

$$
\sigma_{1} \geq \sigma_{2} \geq \ldots \sigma_{n} \geq 0 .
$$

The condition number can be expressed in terms of these singular values,

$$
k(\mathbf{J})=\frac{\sigma_{1}}{\sigma_{n}}
$$

Thus we can place bounds on $\mathbf{f}$ :

$$
\frac{\|\mathbf{n}\|}{\sigma_{1}} \leq\|\mathbf{f}\| \leq \frac{\|\mathbf{n}\|}{\sigma_{n}}
$$

Consider the case of $m=n+1$ tendons controlling an $n$ DoF device. Starting with equation (3) we can write

$$
\mathbf{f}=\mathbf{f}_{\mathbf{e}}+\lambda \hat{\mathbf{h}}
$$

where $f_{e}$ is the vector of minimum norm joint forces due to the external wrench, so $\mathbf{f}_{\mathbf{e}}=\mathbf{J}^{+} \mathbf{w}=\mathbf{J}\left(\mathbf{J}^{T} \mathbf{J}\right)^{-1} \mathbf{w}$. Also, $\hat{h}$ is a unit vector in the nullspace of the Jacobian with all its elements positive, and by definition orthogonal to $f_{e}$. That is,

$$
\begin{aligned}
& \hat{\mathbf{h}}=\left[h_{1}, h_{2}, \ldots h_{m}\right]^{T} \\
& \text { such that } h_{i}>0, i=1, \ldots, m . \\
& \mathbf{J}^{T} \hat{\mathbf{h}}=0 \\
&\|\hat{\mathbf{h}}\|=1, \\
& \hat{\mathbf{h}} \cdot \mathbf{f}_{\mathbf{e}}=0 .
\end{aligned}
$$

The vector $\hat{\mathbf{h}}$ can be calculated from the Jacobian as:

$$
\hat{\mathbf{h}}=\frac{\left(1-\mathbf{J}\left(\mathbf{J}^{T} \mathbf{J}\right)^{-1} \mathbf{J}^{T}\right) \mathbf{z}}{\left\|\left(1-\mathbf{J}\left(\mathbf{J}^{T} \mathbf{J}\right)^{-1} \mathbf{J}^{T}\right) \mathbf{z}\right\|}, \quad z \neq 0
$$

where $\mathbf{z}$ is an arbitrary vector.

From equations (9) and (12)

$$
\|\mathbf{f}\|=\sqrt{\left\|f_{e}\right\|^{2}+\lambda^{2}}
$$

Using equations (15) and (8) we place a lower bound on the tendon forces in terms of the applied wrench and the maximum singular value of the Jacobian.

$$
\Rightarrow\|\mathbf{f}\| \geq\left\|\mathbf{f}_{\mathbf{e}}\right\| \geq \frac{\|\mathbf{w}\|}{\sigma_{1}}
$$

An upper bound on the tendon forces will now be derived. Clearly $\lambda$ can be made arbitrarily large, but it would be preferable to find the minimum value of $\lambda$ that satisfies all the constraints. This procedure will minimize the tendon forces regardless of the external wrench.

Constraint: $f_{i}>0, \quad i=1, \ldots, m$ where $\mathbf{f}=$ $\left[f_{1}, f_{2}, \ldots, f_{m}\right]^{T}$.

$$
\Rightarrow \lambda \geq \frac{-\mathbf{f}_{\mathbf{e}_{i}}}{\hat{\mathbf{h}}_{i},} \quad i=1, \ldots, m
$$

The minimum value of $\lambda$ that satisfies these constraints is:

$$
\lambda_{m}=\max _{i}\left(\frac{-\mathbf{f}_{\mathbf{e}_{i}}}{\hat{\mathbf{h}}_{i}}\right)
$$

The minimum value of $\lambda$ that satisfies all the constraints independent of $f_{e}$ is given by

$$
\lambda^{*}=\max _{\mathbf{f}_{\mathbf{e}}}\left(\lambda_{m}\right)=\max _{\mathbf{f}_{\mathbf{e}, i}} \frac{-\mathbf{f}_{\mathbf{e}_{i}}}{\hat{\mathbf{h}}_{\boldsymbol{i}}}
$$

The magnitude of $f_{\mathbf{e}}$ can be bounded as in equation (8), but the direction of $f_{e}$ can be specified arbitrarily. 
In the worst case $\mathbf{f}_{\mathbf{e}}$ is largest in the direction where the nullspace vector is smallest. Thus,

$$
\lambda^{*}=\frac{\max _{\mathbf{f}_{\mathbf{e}}}\left(-\mathbf{f}_{\mathbf{e}_{i}}\right)}{\min _{i}\left(\boldsymbol{h}_{i}\right)} \leq \frac{\|\mathbf{w}\|}{\sigma_{n} h_{\min }}
$$

where $h_{\min }=\min _{i}\left(\hat{\mathbf{h}}_{i}\right)$, and $\sigma_{n}$ is the minimum singular value.

From equations (15) and (20) an upper bound can be placed on the tendon forces as follows:

$$
\begin{array}{r}
\|\mathbf{f}\| \leq \sqrt{\left\|\mathbf{f}_{\mathbf{e}}\right\|^{2}+\lambda^{* 2}} \\
\Rightarrow\|\mathbf{f}\| \leq \frac{1}{\sigma_{n}} \frac{\sqrt{h_{\min }^{2}+1}}{h_{\min }}\|\mathbf{w}\|
\end{array}
$$

Similarly, if we apply a small perturbation $\delta$ w to the system the resulting tendon force is bounded by:

$$
\|\delta \mathbf{f}\| \leq \frac{1}{\sigma_{n}} \frac{\sqrt{h_{\min }^{2}+1}}{h_{\min }}\|\delta \mathbf{w}\|
$$

From the lower bound of equation (16) we have

$$
1 /\|\mathbf{f}\| \leq \sigma_{1} /\|\mathbf{w}\|
$$

Combining equations (23) and (22) we arrive at the final result,

$$
\frac{\|\delta \mathbf{f}\|}{\|\mathbf{f}\|} \leq k(\mathbf{J}) \frac{\sqrt{h_{\min }^{2}+1}}{h_{\min }} \frac{\|\delta \mathbf{w}\|}{\|\mathbf{w}\|}
$$

where $k(J)=\sigma_{1} / \sigma_{n}$ is the condition number of the Jacobian.

The quantity $k(J) \frac{\sqrt{h_{\min }^{2}+1}}{h_{\min }}$ plays the same role as condition number for linear systems. In fact this measure incorporates the condition number itself. The maximum value of $h_{\min }$ subject to constraint (10) and (12) occurs when all the elements of $\hat{\mathbf{h}}$ are equal to $1 / \sqrt{m}$. Thus the local tendon dexterity measure $T D_{l}$ is defined as:

$$
T D_{l} \equiv \begin{cases}\sqrt{m+1} \frac{h_{\text {min }}}{k(J) \sqrt{h_{\text {min }}^{2}+1}} & \text { if } h_{\min } \geq 0 \\ 0 & \text { otherwise }\end{cases}
$$

This measure is normalized such that $0 \leq T D_{l} \leq 1$. It is zero when the Jacobian is singular or when condition (10) is violated. This will be referred to as an extended singularity as it has all the properties of regular singularities including large actuator forces for small external wrenches. The tendon dexterity is one when the linear system is isotropic and when the nullspace vector is uniform in all directions. That is $\hat{h}=1 / \sqrt{m}[1,1, \ldots, 1]^{T}$, or stated in terms of condition (11) the columns of the Jacobian must sum to zero. This will be referred to as extended isotropy.

As a bonus, equation (21) gives an upper bound on tendon forces which in itself can be used as a dexterity measure. To keep the measure finite, the reciprocal will be taken and it will be scaled, thus a second dexterity measure follows:

$$
T F_{l} \equiv \begin{cases}\sqrt{m+1} \frac{\sigma_{n} h_{\min }}{\sqrt{h_{\min }^{2}+1}} & \text { if } h_{\min } \geq 0 \\ 0 & \text { otherwise }\end{cases}
$$

This has the same physical significance as the minimum singular value measure of linear systems. $T F_{l}$ is zero at an extended singularity. Maximizing this quantity effectively minimizes the tendon forces in a particular configuration.

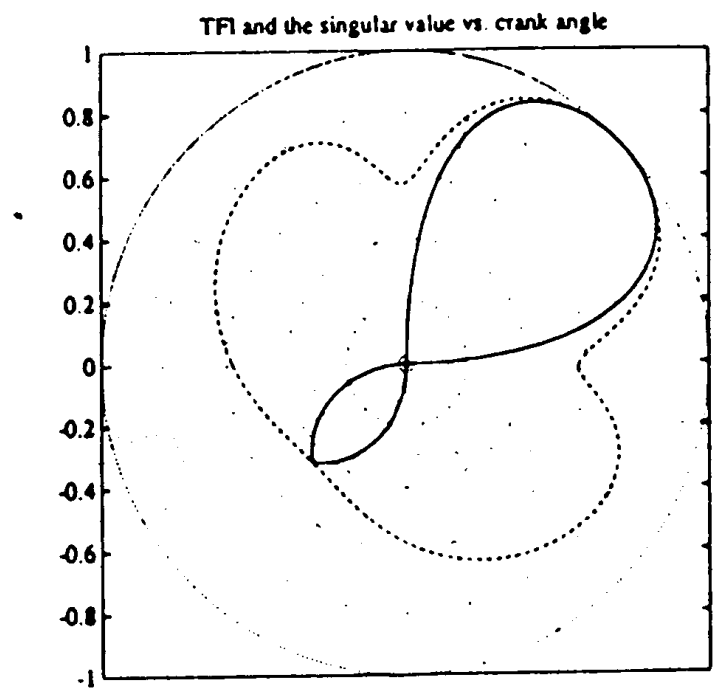

Figure 2: Dexterity of a tendon actuated crank. The solid line plots $T F_{l}$ and the dashed line plots the singular value $\left(F_{l}\right)$. In certain regions $T F_{l}$ is zero, and the crank can not be moved in both directions with tendons. If pistons were used then the mechanism would always be controllable.

As an example figure 2 plots the singular value and $T F_{I}$ for the tendon-crank system of figure $1(b)$. Here $n=1$ so there is only one singular value, thus condition number measures do not apply. Notice there are regions for which $T F_{l}$ is zero where the crank cannot be moved in all directions by tendons. If pistons were used then these singularities would not exist as shown by the nonzero singular value. 


\section{Force mapping}

In this section the tendon dexterity will be interpreted in relation to the force mapping characteristics. For ordinary mechanisms, if the joint forces exist in the unit sphere $\|f\| \leq 1$ then the external wrench maps to the ellipsoid $\mathbf{w}^{T}\left(J^{T} J\right)^{-1} \mathbf{w} \leq 1$. It has principal axes in the direction of the eigenvectors of $\left(J^{T} J\right)^{-1}$ and magnitudes equal to the corresponding singular value.

Since tendons impose nonlinear constraints the unit sphere will no longer map to an ellipsoid. It will map to a distorted shape with radius $r=r(w, J)$. From equations (3) and (18),

$$
r=\frac{1}{\sqrt{\hat{\mathbf{w}}^{T}\left(\mathbf{J}^{T} \mathbf{J}\right)^{-1} \hat{\mathbf{w}}+\lambda_{m}^{2}}}
$$

where $\hat{w}$ is an external wrench of unit magnitude.

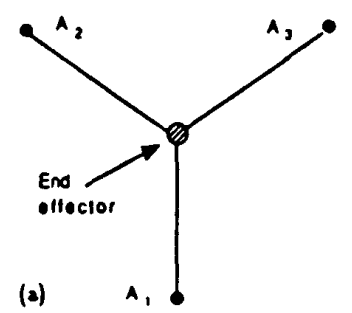

(b)

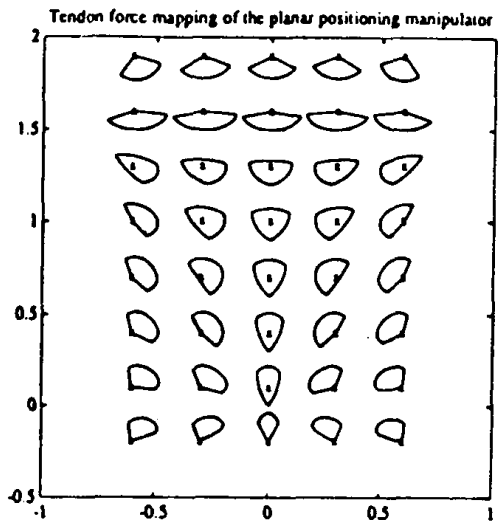

Figure 3: Tendon actuated parallel positioning manipulator (a). The force mapping at various points (b). The origin of each mapping is marked with an ' $x$ '. Outside the indicated triangle the dexterity is zero.

Consider the tendon actuated planar parallel manipulator of figure 3.a. It is possible to manipulate objects within the triangle $A_{1} A_{2} A_{3}$. The force mapping at various points is shown in figure 3.b. Directions with large radius with respect to the origin (' $x$ ') are able to transmit large forces in the corresponding direction. Outside the triangle there will be directions for which it is impossible to produce forces. At the centroid of the triangle the manipulator is extended isotropic, however the force mapping is not circular as one might expect.

Based on the analysis of the previous section,

$$
\sigma_{1} \geq r_{\max } \geq r \geq r_{\min } \geq \frac{\sigma_{n} h_{\min }}{\sqrt{h_{\min }^{2}+1}}
$$

Thus

$$
\frac{1}{\sqrt{m+1}} T D_{l} \leq \frac{r_{\min }}{r_{\max }}
$$

Notice that the tendon dexterity is not proportional to the ratio of minimum to maximum radius but does provide a lower bound. This is a result of the simplifications of the previous section where the terms fe and $\lambda$ were maximized (minimized) separately even though they are dependent. As a comparison, for bi-directional actuators $D_{l}=\frac{r_{\operatorname{mik}}}{r_{\max }}$ exactly.

\section{Conclusion}

We have developed a dexterity measure applicable to a class of tendon-driven mechanisms which is formed by bodies held under force closure by a set of tendons; and for which conventional dexterity measures lead to erroneous conclusions.

The figure 4 illustrates some of these mechanisms. The spherical case is drawn from (Hayward 1990).

\section{References}

Hayward, V. (1990) "An analysis of redundant manipulators from several viewpoints". Robots with redundancy: design, sensing and control, NATO Series, A. Bejczy (Ed.), Springer Verlag, In press.

Jacobsen, S. C., Wood, J. E., Knutti, D. F., Biggers, K. B., (1984) "The UTAH/M.I.T. dextrous hand: work in progress". The Int. Journal of Robotics Research, Vol. 3, No. 4, pp. 21-49.

Kerr, J., Roth, B., (1986a) "Analysis of multifingered hands". Int. Journal of Robotics Research, Vol. 4, No. 4, pp. 3-17.

Markenscoff, X., and Papadimitriou, C. H., (1989) "Optimum grip of a polygon". The Int. Journal of Robotics Research, Vol. 8, No. 2, pp.17-29.

Lakshminarayana, K., (1978) "Mechanics of form closure". ASME Design Engineering Technical Conference Minneapolis, Minn., Paper No. 78-DET-32 
Salisbury, J. K., Townsend, W., Eberman, B., DiPietro, D., (1988) "Preliminary design of a whole-arm manipulation system (WAMs)". IEEE Int. Conf. on Robotics and Automation, Vol. 1, pp. 254-260.

Tsuda, M., Nakamura, Y., Higushi, T. (1990) "HighSpeed Digital Controller for Magentic Servo Levitation of Robot Mechanisms." in Experimental Robotics 1, V. Hayward, O. Khatib (Eds.), Lecture Notes in Control and Information Science, 139, Springer Verlag.

Tsuda, M., Nakamura, Y., Higuchi, T., (1989) "Design and control of magnetic servo levitation". Proc. 20th Int. Symp. on Industrial Robots.

Vertut, J., Marchal, P., Debrie, G., Petit, M., Francois, D., Coiffet, P., (1976) "The MA 23 bilateral servomanipulator system". Proc. of the 24th Conf. on Remote Systems Technology, Washington. pp. 175-187.

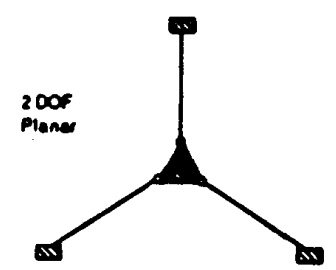

3006
Orionialion

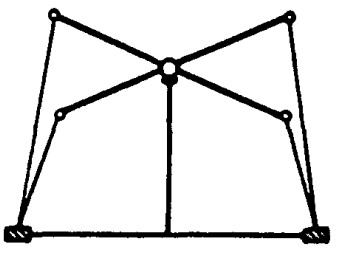

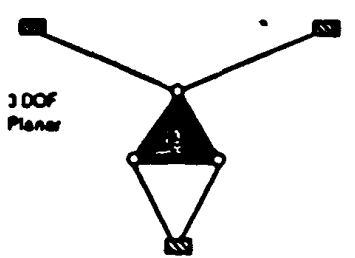
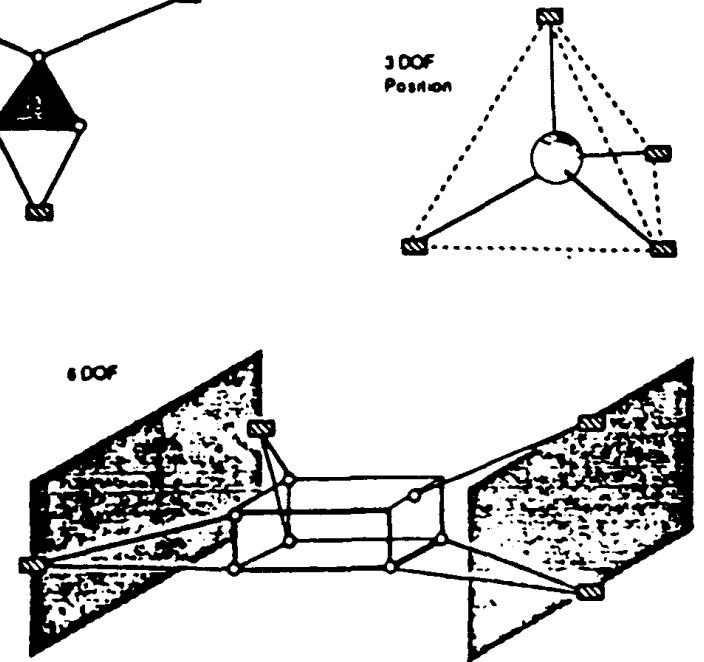

Figure 4: Some parallel mechanisms actuated by the mininum number of tendons. 